\title{
Die DEGUM mischt sich ein
}

Liebe Kolleginnen und Kollegen, liebe DEGUM-Mitglieder,

im März 2014 erschien im Deutschen Ärzteblatt ein ausführlicher Artikel zur Diagnostik von Lungenerkrankungen (DÄB 2014; 111(11): 181-7 bzw. DOI: 10.3238/ arzte-bl.2014.0181). Die dort als Schlussfolgerung vorgenommene Empfehlung einer angepassten Auswahl der diagnostischen Verfahren Röntgen, CT und MRT, insbesondere auch zur Vermeidung einer Überdiagnostik, ließ nicht nur bei Ultraschall-Unkundigen den Eindruck entstehen, dass anderen diagnostischen Verfahrenen bei diesen Erkrankungen keinerlei Bedeutung zukommt und diese damit auch keine Berechtigung hätten.

Dass dies für den Ultraschall nicht gilt, betonte Dr. J.-C. Kämmer, Leiter des Arbeitskreises Thoraxsonografie der DEGUM in seinem Leserbrief an das Deutsche Ärzteblatt (DÄB 2014; 111(21): 376 bzw. DOI: 10.3238 /arzte-bl.2014.0376a). So ist der Ultraschall in der Intensiv- und Notfallmedizin von Lungen- und Thoraxerkrankungen nicht mehr wegzudenken und wird er bei Kindern als primäres diagnostisches Verfahren am Thorax empfohlen. In ihrem Schlusswort bestätigen die Autoren die von J.-C. Kämmer gemachten Einwände und erweitern ihre im Artikel getroffenen Aussagen dahingehend, dass der Ultraschall frühzeitig in der Diagnostik eingesetzt werden sollte, um weitergehende Verfahren entweder zu vermeiden oder gezielt zu indizieren (DOI: 10.3238/ arzte-bl.2014.0376b).

Diesen Weg des „Stellungnehmens“ zu Print- und anderen Veröffentlichungen, des „Einmischens“ in Diskussionen, wenn es um Ultraschall geht oder, wie oben, nicht geht, obwohl Ultraschall ein Thema sein sollte, wollen wir in Zukunft intensivieren. Die Expertise, die die DEGUM und ihre Mitglieder zum Ultraschall haben und die von anderen Fachgesellschaften und Gremien anerkannt wird, wollen wir wahrnehmbar in die öffentliche und medizinische Diskussion einbringen. Helfen Sie uns dabei! Informieren Sie die Geschäftsstelle (geschaeftsstelle@degum. de), wenn Sie der Meinung sind, dass Publikationen oder anderweitige Veröffentlichungen unter dem Aspekt des Ultraschalls kommentiert werden sollten. Der Vorstand, Frau Dr. Jagota und die Mitarbeiterinnen der Geschäftsstelle werden die Sektionen und Arbeitskreise um Stellungnahmen bitten und diese in Ihrem und dem Namen der DEGUM an den Adressaten übermitteln. Parallel dazu leiten wir Ihre Zuarbeit an die Pressestelle der DEGUM weiter, die diese in Abhängigkeit des Publikationsmediums redigiert und nach Ihrer Zustimmung in den Presseverteiler gibt und auf unserer Homepage veröffentlicht.

Die deutliche Ausweitung dieser „externen Präsenz" für den Ultraschall - neben oben Genanntem z. B. in Form von Ständen auf den Jahrestagungen anderer medizinischer Fachgesellschaften und dem konsequenten Einbringen unserer Expertise bei Anfragen medizinpolitischer Gremien wie dem Gemeinsamen Bundesausschuss oder dem IQWIG - wäre ohne die Neustrukturierung der DEGUM-Verwaltung nicht möglich. Durch die effizientere
Bearbeitung von Kursanträgen und Organisation von Gremientagungen/-sitzungen der Sektionen und Arbeitskreise mithilfe des neuen Kursportals und dem seit kurzem neu installierten Veranstaltungsportal auf der Homepage der UltraschallAkademie (www.ultraschall-akademie. de) konnten wir die dafür erforderlichen Ressourcen und Kapazitäten bei den Mitarbeiterinnen der Geschäftsstelle freisetzen.

An dieser Stelle heißen wir Frau Sabrina Reuter als neue Mitarbeiterin der Geschäftsstelle seit 1.9.2014 herzlich willkommen. Ihr Aufgabenbereich wird, gemeinsam mit Frau Dr. Jagota, das Wissenschaftsreferat sein. Einer ihrer ersten Aufgaben ist die Erfassung aller ultraschallrelevanten Leitlinien-Projekte im Leitlinienmodul der neuen Mitgliederdatenbank. Sie wird mit allen Leitliniendelegierten der DEGUM Kontakt aufnehmen und nach Möglichkeiten einer besseren Abbildung des Ultraschalls in der jeweiligen Leitlinie fragen. Mittelfristig will der DEGUM-Vorstand gezielt Wissenschaftsprojekte fördern und / oder initiieren, die die für Leitlinien essenzielle, im Bereich des Ultraschalls jedoch häufig fehlende oder insuffiziente Evidenz helfen zu generieren.

\section{MG, Sekretär \\ AJ, Leiterin Geschäftsstelle}

